\title{
Erratum to: FragClust and TestClust, two informatics tools for chemical structure hierarchical clustering analysis applied to lipidomics. The example of Alzheimer's disease
}

\author{
Francesca Di Gaudio $^{1} \cdot$ Sergio Indelicato $^{1} \cdot$ Roberto Monastero $^{2} \cdot$ Grazia Ida Altieri $^{3}$ • \\ Francesca Fayer $^{3}$ • Ornella Palesano ${ }^{3}$ - Manuela Fontana ${ }^{1}$ • Angelo B. Cefalu ${ }^{1}$. \\ Massimiliano Greco ${ }^{3}$ - David Bongiorno ${ }^{4}$ - Serena Indelicato ${ }^{4}$. Angela Aronica ${ }^{2}$. \\ Davide Noto $^{3} \cdot$ Maurizio R. Averna ${ }^{3}$
}

Received: 9 February 2016 / Accepted: 9 February 2016/Published online: 19 February 2016

(C) Springer-Verlag Berlin Heidelberg 2016

Erratum to: Analytical and Bioanalytical Chemistry

DOI: 10.1007/s00216-015-9229-6

The authors would like to call the reader's attention to the fact that unfortunately the acknowledgement section was not mentioned in the article.

The sentence should read:

Acknowledgments

The ZAP 10-year follow-up data collection was supported by a grant from the Italian Ministry of Health to R.M. (project for young researcher 2007, GR-2007-686973).

Francesca Di Gaudio and Sergio Indelicato contributed equally to this work.

Davide Noto and Maurizio R Averna contributed equally to this work.

The online version of the original article can be found at http://dx.doi.org/ 10.1007/s00216-015-9229-6.

Maurizio R. Averna

maurizio.averna@unipa.it

1 Mass Spectrometry Laboratory for Clinical Risk and Quality Control, A.O.U.P. "P. Giaccone", University of Palermo, Via del Vespro 129, 90127 Palermo, Italy

2 Department of Experimental Biomedicine and Clinical Neurosciences, University of Palermo, Via del Vespro 129, 90127 Palermo, Italy
Department of Biomedicine Internal Medicine and Medical Specialties, Division of Internal Medicine and Genetic Dyslipidemias, University of Palermo, Via del Vespro 141, 90127 Palermo, Italy

4 Dipartimento di Scienze e Tecnologie Biologiche Chimiche e Farmaceutiche(STEBICEF), University of Palermo, Viale delle Scienze, Ed. 16, 90128 Palermo, Italy 